\title{
Respuesta de Glycine max a Azospirillum lipoferum y Rhizobium etli a dosis $50 \%$ de fertilizante nitrogenado
}

\author{
Response of Glycine max to Azospirillum lipoferum y Rhizobium etli at $50 \%$ dose of nitrogen \\ fertilizer
}

\author{
Santoyo-Pizano Gustavo ${ }^{1}$, Ignacio-Cruz Juan Luis ${ }^{1}$, Vargas-Hernández Martha Elizabeth ${ }^{1}$, \\ Gallegos-Morales Gabriel $^{2}$, Sánchez-Yáñez Juan Manuel ${ }^{1 *}$
}

\begin{tabular}{|c|c|}
\hline Datos del Articulo & Resumen \\
\hline 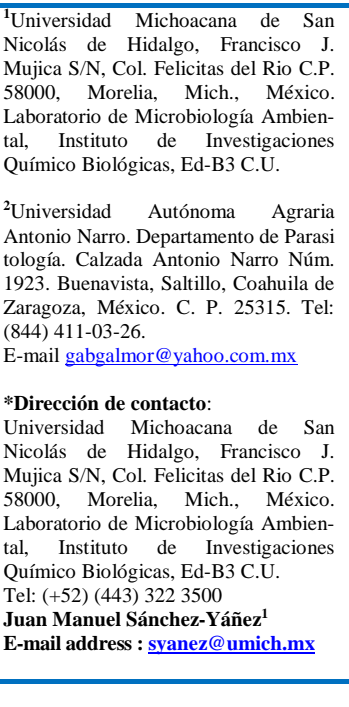 & $\begin{array}{l}\text { La producción de Glycine max (soya) requiere fertilizante nitrogenado (FENI), como el } \mathrm{NH}_{4} \mathrm{NO}_{3} \text { (nitrato de amo- } \\
\text { nio) para suplir la demanda de } \mathrm{N} \text { (nitrógeno), el cual aplicado en exceso provoca pérdida de productividad del } \\
\text { suelo. Una alternativa de solución para este problema es la reducción y optimización de la dosis de FENI, mediante } \\
\text { géneros y especies de bacterias promotoras de crecimiento vegetal (BAPOCEVE) como: Azospirillum lipoferum y } \\
\text { Rhizobium etli. El objetivo de esta investigación fue analizar la respuesta de G. max a la inoculación con A. lipo- } \\
\text { ferum y } R \text {. etli a la dosis } 50 \% \text { del FENI. El ensayo se realizó en un invernadero, bajo un diseño experimental con } 5 \\
\text { tratamientos mediante las variables-respuesta: fenología de la parte aérea y radical, altura de planta (AP), longitud } \\
\text { de raíz (LR) y de la biomasa, peso fresco y seco aéreo/radical (PFA/PFR)/(PSA/PSR) a plántula y floración. Los } \\
\text { datos experimentales se analizaron por ANOVA/Tukey HSD p<0.05\%. Los resultados mostraron a nivel de plántu- } \\
\text { la una respuesta de G. max a la doble acción con A. lipoferum y } R \text {. etli al } 50 \% \text { del FENI con } 0.90 \mathrm{~g} \text { de PSA, valor } \\
\text { numérico estadísticamente diferente comparado con los } 0.16 \mathrm{~g} \text { de PSA de } G \text {. max sin inocular alimentada con una } \\
\text { solución mineral y el FENI al } 100 \% \text { o control relativo (CR). A floración } G \text {. max con ambos géneros de BAPOCE- } \\
\text { VE se registró } 3.17 \mathrm{~g} \text { de PSR, valor numérico estadísticamente diferente a los } 1.79 \mathrm{~g} \text { de PSR de } G \text {. max usado como } \\
\text { CR. Lo anterior sugiere que } G \text {. max tratado con A. lipoferum y } R \text {. etli convirtieron sus exudados radicales en fi- } \\
\text { tohormonas, para mejorar la absorción radical del N y optimizar el FENI a la dosis } 50 \% \text {, con un sano crecimiento y } \\
\text { evitar al mismo tiempo la pérdida de productividad del suelo. }\end{array}$ \\
\hline \multirow{2}{*}{$\begin{array}{l}\text { Suelo, } \\
\text { FENI, } \\
\text { Glycine max, } \\
\text { Azospirillum lipoferum, } \\
\text { Rhizobium etli, } \\
\text { exudados radicales, } \\
\text { fitohormonas. }\end{array}$} & (C) 2018. Journal of the Selva Andina Research Society. Bolivia. Todos los derechos reservados. \\
\hline & Abstract \\
\hline $\begin{array}{l}\text { J. Selva Andina Res. Soc. } \\
2018 ; 9(2): 96-103 .\end{array}$ & \multirow{3}{*}{$\begin{array}{l}\text { The production of Glycine max (soybean) demands nitrogenous fertilizer (NIFE), as a } \mathrm{NH}_{4} \mathrm{NO}_{3} \text { (ammonium nitrate) } \\
\text { to supply its } \mathrm{N} \text { demand, which when is applied in excess causes lost soils productivity. An alternative for this } \\
\text { problem is to reduce and to optimize NIFE, with inoculants based in genus and species of plant growth promoting } \\
\text { bacteria (PGPB). The aim of this research was to analyze G. max response to Azospirillum lipoferum and Rhizobi- } \\
\text { um etli at } 50 \% \text { dose of NIFE. In that sense experimental was conducting by } 5 \text { treatments with NIFE at } 100 \% \text { and } \\
50 \% \text { doses for G. max inoculated with PGPB, response was based on its phenology: plant height (PH) and root } \\
\text { length (RL) and its biomass: shoot fresh weight (SFW), root fresh weight (RFW), shoot dry weight (SDW), root dry } \\
\text { weight (RDW). Experimental data were analyzed by ANOVA and Tukey HSD p<0.05\%. Results indicated a posi- } \\
\text { tive respond of G. max with A. lipoferum and } R \text {. etli at seedling level was registered } 0.90 \mathrm{~g} \text { of SDW, this numerical }\end{array}$} \\
\hline $\begin{array}{l}\text { Historial del artículo. } \\
\text { Recibido febrero, } 2018 . \\
\text { Devuelto junio } 2018 \\
\text { Aceptado junio, } 2018 . \\
\text { Disponible en línea, agosto, } 2018 .\end{array}$ & \\
\hline $\begin{array}{c}\text { Editado por: } \\
\text { Selva Andina } \\
\text { Research Society }\end{array}$ & \\
\hline
\end{tabular}


value was statistically different than $0.16 \mathrm{~g}$ of SDW of G. max not inoculating, fed with mineral solution with $100 \%$ of NIFE dose, used as relative control (RC). At level of flowering G. max responded to A. lipoferum and R. etli registered $3.17 \mathrm{~g}$ of RFW this numerical value was statistically different compared to $1.79 \mathrm{~g}$ of RFW of $G$. $\max$ or RC. This data suggests that the positive respond of G. $\max$ was due to both PGPB able to transform radical exudates in phytohormones to improve radical absorption of $\mathrm{N}$ and optimize NIFE reduced at $50 \%$ dose, showing health growing and at the same preventing soil`s lost productivity.

(C) 2018. Journal of the Selva Andina Research Society. Bolivia. All rights reserved.

\section{Introducción}

La leguminosa Glycine $\max$ (soya), tiene un alto valor nutricional, en México la zona relacionada, su producción anual asciende a 400000 ton/ha (SIAPSAGARPA 2016), generando una demanda de $\mathrm{N}$ (nitrógeno) como fertilizante nitrogenado (FENI), $\mathrm{NH}_{4} \mathrm{NO}_{3}$ (nitrato de amonio), aplicado en exceso provoca la rápida degradación de la materia orgánica del suelo, con la consecuente disminución de la productividad (Isbell et al. 2013). En el suelo una alternativa de solución que evite la hiperfertilización nitrogenada, es la inoculación de G. max con géneros de bacterias o microorganismos promotores de crecimiento vegetal (BAPOCEVE) o MICROCEVE) (Armenta-Bojórquez et al. 2010): como el género fijador simbiótico de $\mathrm{N}_{2}$ : Bradyrhizobium japonicum que en $G$. max permite un crecimiento sano a dosis regulada de FENI, no obstante, en función del origen de B. japonicum se inducen nódulos, que no son necesariamente eficaces para incorporar el $\mathrm{N}_{2}$ como una fuente sustituta del FENI, para asegurar un sano crecimiento de G. max, en cuyo caso se recomienda inocular un Rhizobium etli del grupo conocido como de inoculación cruzada en las leguminosas, y que también puede ser benéfico para $G$. max, en combinación con un fijador libre de $\mathrm{N}_{2}$ libre como: Azospirillum lipoferum (Loredo-Osti et al. 2004) u otra clase de MICROCEVE, como es el caso de géneros y especies de hongos micorrizicos versículo arbusculares (HMVA) que favorezcan una mayor absorción del FENI, e incluso del fertilizante del fosforo (P) como $\mathrm{PO}_{4}^{-3}$ (FEPO) en especial cuando se reduce al $50 \%$, puesto que los exudados radicales de G. max contienen suficientes fuentes de $\mathrm{C}$ (carbono) y $\mathrm{N}$ orgánico, que pueden ser transformados en sustancias promotoras de crecimiento vegetal (SUPOCEVE) o fitohormonas (Matiru \& Dakora 2004), compuestos que inducen una mayor absorción radical del $\mathrm{N}$ y con ello la optimización del FENI y/o FEPO reducido al 50\%, sin afectar el sano crecimiento vegetal. Con base en lo anterior se han realizado investigaciones para mejorar la producción de G. max mediante el empleo de inoculantes a base de BAPOCEVE o MICROCEVE, bajo un esquema de agricultura sustentable, que explota el recurso suelo como el vegetal sin prejuicio de ambos o del ambiente (Armenta-Bojórquez et al. 2010). Como lo prueban las siguientes investigaciones: Uhrich \& Benintende 2005 analizaron la respuesta positiva de G. max a B. japonicum y Azospirillum brasilense a nivel de campo. Los resultados mostraron un incremento de un $29 \%$ en el peso seco radical (PSR), en comparación con G. max sin inocular con BAPOCEVE a la dosis de FENI al 100\% o control relativo (CR). De la misma Hernández 2008 reporto la respuesta positiva de $G$. max a la inoculación con Glomus hoi like y B. japonicum a dosis reducida de FENI y FEPO. Los resultados 
mostraron que ambos microorganismos mejoraron absorción del $\mathrm{N}$ y $\mathrm{P}$, con un rendimiento mayor al comparado con G. max sin inocular y con las dosis recomendada de $\mathrm{N}$ y $\mathrm{P}$. Mientras que Benintende et al. 2010 reportaron la respuesta positiva de G. max a B. japonicum y A. brasilense a dosis regulada de FENI. Los resultados mostraron $G$. max con ambas BAPOCEVE incrementaron hasta un 30\% de crecimiento vegetativo, en contraste con G. $\max$ sin inocular usado como CR a la dosis $100 \%$ del FENI. En tanto que Corbera-Gorotiza \& Nápoles-García 2013 reportaron la respuesta positiva de G. max tratado con Bradyrhizobium elkanii y un HMVA a dosis regulada del FENI y FEPO. Encontraron un incremento en el rendimiento de G. max inoculado con los MICROCEVE comparado con G. max alimentada exclusivamente con la dosis recomendada del FENI y FEPO. Sin embargo los HMVA tienen algunos inconvenientes para la inoculación de semillas de G. max, debido a que pierden rápidamente la viabilidad contra factores bióticos y abióticos. Por lo anterior podría ser más útil emplear géneros y especies de BAPOCEVE, con especial resistencia a factores ambientales que limitan la actividad positiva de otro de MICROCEVE como los HMVA, para la eficaz absorción y optimización radical del FENI en específico cuando se reduce al 50\% de acuerdo con la literatura disponible (Uhrich \& Benintende 2005, Hernández 2008). Por lo que el objetivo de este trabajo fue analizar la respuesta de Glycine max a, A. lipoferum, Azospirillum lipoferum y Rhizobium etli a la dosis $50 \%$ de FENI.

\section{Materiales y métodos}

El experimento se realizó en el invernadero de $10 \mathrm{x}$ $4 \mathrm{~m}^{2}$ del Laboratorio de Microbiología Ambiental del Instituto de Investigaciones Químico Biológicas de la UMSNH, Morelia, Mich, México. La tempera- tura promedio fue de $23.2^{\circ} \mathrm{C}$, luminosidad de 450 $\mathrm{mol} \mathrm{m} \mathrm{m}^{-2} \cdot \mathrm{s}^{-1}$, humedad relativa de $67 \%$, se regulo mediante un sistema automático de control ambos factores ambientales (Matallana \& Montero 2001). Para este ensayo se utilizó un suelo arcilloso de tipo Vertisol con un historial de 20 año en producción de trigo/garbanzo un pobre en materia orgánica y $\mathrm{N}$ mineral, que se solarizó para minimizar el problema de plagas y enfermedades, posteriormente se tamizó con una malla del No 20 y en el invernadero se usaron insecticidas a base de extracto vegetales de canela y ajo para el control fitosanitario (Duke 1990).

\section{Figura 1. Diagrama de una jarra de Leonard (García-González et al. 2005).}

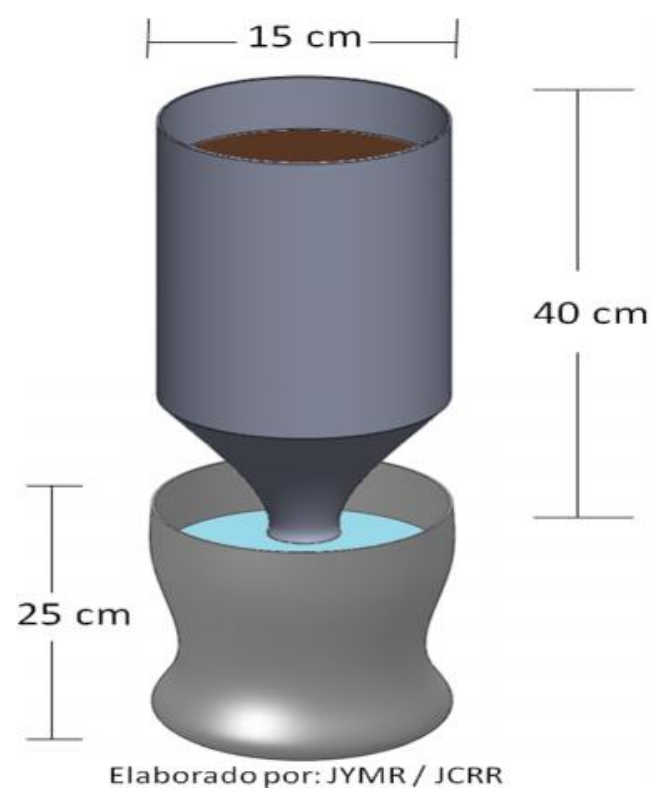

Las semillas de G. max fueron donadas por la Secretaria y Agricultura y Recursos Hidráulicos (SIAPSAGARPA 2016), estas semillas se desinfectaron con $\mathrm{NaClO}$ (Hipoclorito de sodio) al 3\% (v/v) por 5 min y se enjuagaron 6 veces con agua estéril, después se desinfectaron con alcohol al $70 \%(\mathrm{v} / \mathrm{v})$ por 5 min y se lavaron 5 veces con agua estéril (GarcíaGonzález et al. 2005). Posteriormente en bolsa de 
plástico de $250 \mathrm{~g}$ las semillas de G. max se inocularon con $2.0 \mathrm{~mL}$ de A. lipoferum, $R$. etli de manera individual y/o en mezcla, ambos casos se dejaron por 24 h. A. lipoferum se aisló de raíces de Zea mays var mexicana (teocintle) en agar NFM (nitrogen free medium) (Döbereiner \& Day 1974) mientras que $R$. etli se recuperó de nódulos de Medicago sp en agar manitol rojo congo (Peña-Cabriales \& Alexander 1981, Sánchez-Yáñez 2007). Ambos géneros de BAPOCEVE pertenecen a la colección del laboratorio de Microbiología Ambiental del IIQB-UMSNH, en Morelia, Mich, México. Para este ensayo se seleccionó un $1 \mathrm{~kg}$ de suelo degradado y compactado con un historial agrícola de 20 años de cultivo intensivo de gramíneas (maíz-trigo, maíz-cebada), de textura arcillosa con un bajo contenido de materia orgánica de $1.5 \%$ y pobre $\mathrm{N}$ orgánico pobre con $39 \mathrm{~kg} / \mathrm{ha}$, con un $\mathrm{pH} 6.7$ (ligeramente acido), ubicado a los $19^{\circ} 39^{\prime} 27^{\prime \prime}$ de latitud norte y $101^{\circ} 19^{\prime} 59^{\prime}$ de longitud oeste, con temperatura media anual de $17.3{ }^{\circ} \mathrm{C}$ y precipitación anual de $796.4 \mathrm{~mm}$, en un terreno agrícola denominado "La Cajita" de la Tenencia Zapata del Municipio de Morelia, Mich., México, ubicado en el km 5 de la carretera Morelia-Pátzcuaro. Este kg de suelo se colocó en la parte superior del sistema hidropónico conocido como jarra de Leonard mostrado en la figura 1, ahí se sembraron 4 semillas de G. max, mientras que el agua, o la SOMI con el FENI reducido al 50\%, que se colocó en la parte inferior, ambas partes se conectaron por una tira de algodón de $15.0 \mathrm{~cm}$. La SOMI con el FENI para alimentar $G$. max se preparó con la siguiente composición química (g/L): $\mathrm{NH}_{4} \mathrm{NO}_{3}$ 12.0, $\mathrm{KH}_{2} \mathrm{PO}_{4} 3.0, \mathrm{~K}_{2} \mathrm{HPO}_{4} 3.5$, $\mathrm{MgSO}_{4} 1.5, \mathrm{CaCl}_{2} 0.1, \mathrm{FeSO}_{4} 0.5 \mathrm{~mL} / \mathrm{L}$, y un 1.0 $\mathrm{ml} / \mathrm{L}$ de la solución de oligoelementos: ( $\mathrm{g} / \mathrm{L})$, $\mathrm{H}_{3} \mathrm{BO}_{3} 2.86, \mathrm{ZnSO}_{4} 7 \mathrm{H}_{2} \mathrm{O} \quad 0.22, \mathrm{MnCl}_{2} 7 \mathrm{H}_{2} \mathrm{O} 1.81$, $\mathrm{K}_{2} \mathrm{MnO}_{4}$ 0.09, a pH ajustado a 7.0, en agua destilada, mientras que el $\mathrm{NH}_{4} \mathrm{NO}_{3}$ o FENI se redujo al $50 \%$ equivalente a $6 \mathrm{~g} / \mathrm{L}$ (García-González et al. 2005).

En la Tabla 1 se indica el diseño experimental con 5 tratamientos y 7 repeticiones para analizar la respuesta de G. max a la inoculación con A. lipoferum y $R$. etli a nivel de plántula y floración con la dosis del FENI reducido al 50\%, mediante las variablesrespuesta basados en la fenología: la altura de planta (AP), longitud de raíz (LR), y en la biomasa: el peso fresco aéreo (PFA) y radical (PFR), para el peso seco aéreo (PSA) y radical (PSR), G. max se secó en horno a $40{ }^{\circ} \mathrm{C} / 48$ h (Sánchez-Yáñez 2007). Los datos experimentales fueron sometidos al análisis de varianza ANOVA mediante la prueba comparativa de medias de Tukey HSD P $<0.05 \%$ (GarcíaVillalpando et al. 2001).

Tabla 1 Diseño experimental para analizar la respuesta de Glycine max a la inoculación con Azospirillum lipoferum y Rhizobium etli dosis $50 \%$ del fertilizante nitrogenado

\begin{tabular}{cccccc}
\hline$*$ Tratamiento & Glycine max & Azospirillum lipoferum & Rhizobium etli & Agua & ${\text { SOMI con } \mathbf{N H}_{4} \mathrm{NO}_{3}}$ \\
\hline Control absoluto & + & - & - & + & - \\
Control relativo & + & - & - & - & $+100 \%$ \\
1 & + & + & - & - & $+50 \%$ \\
2 & + & - & + & - & $+50 \%$ \\
3 & + & + & + & - & $+50 \%$ \\
\hline
\end{tabular}




\section{Resultados}

En la Tabla 2, se muestra la respuesta de G. max la doble inoculación con A. lipoferum, $R$. etli a dosis $50 \%$ a nivel de plántula, que alcanzó $38.7 \mathrm{~cm}$ de altura de planta (AP) y $42.2 \mathrm{~cm}$ de longitud radical (LR); ambos valores numéricos fueron estadísticamente diferentes comparados con los $30.1 \mathrm{~cm}$ de AP y los $15.7 \mathrm{~cm}$ de LR en G. max sin inocular alimentada con la dosis $100 \%$ del FENI, referido como CR. En relación a la biomasa de G. max inoculada con la mezcla de amabas BAPOCEVE registró 5.62 g de PFA y 1.96 g de PFR; estos valores numéricos tuvieron diferencia estadística respecto a los $1.26 \mathrm{~g}$ de PFA y $0.66 \mathrm{~g}$ de PFR en G. max usado como CR. En tanto que G. max con A. lipoferum y R. etli registro $1.80 \mathrm{~g}$ de PSA y $0.75 \mathrm{~g}$ de PSR ambos valores numéricos tuvieron con diferencia estadística comparado con los $0.63 \mathrm{~g}$ de PSA y $0.09 \mathrm{~g}$ de PSR de $G$. max empleado como CR.

En la Tabla 3, se muestra la respuesta de G. max a la inoculación con A. lipoferum y $R$. etli a la dosis $50 \%$ a floración, donde G. max alcanzó $44.7 \mathrm{~cm}$ de AP y $33.8 \mathrm{~cm}$ de LR, ambos valores numéricos tuvieron diferencia estadística comparado con los $39.3 \mathrm{~cm}$ de AP y $25.7 \mathrm{~cm}$ de LR de G. max sin inocular alimentada con la dosis $100 \%$ de FENI usado como CR

Tabla 2 Respuesta de Glycine max a la inoculación con Azospirillum lipoferum y Rhizobium etli a dosis $50 \%$ del fertilizante nitrogenado a nivel de plántula

\begin{tabular}{|c|c|c|c|c|c|c|}
\hline \multirow{2}{*}{ *Glycine max en suelo } & \multirow{2}{*}{$\mathbf{A P}(\mathbf{c m})$} & \multirow{2}{*}{$\mathbf{L R}(\mathbf{c m})$} & \multicolumn{2}{|c|}{ Peso fresco $(\mathrm{g})$} & \multicolumn{2}{|c|}{ Peso seco $(\mathrm{g})$} \\
\hline & & & Aéreo & Radical & Aéreo & Radical \\
\hline Irrigado con agua (Control Absoluto) & $26.3^{\mathrm{e}^{* *}}$ & $15.3^{\mathrm{d}}$ & $1.20^{\mathrm{e}}$ & $0.64^{\mathrm{d}}$ & $0.31^{\mathrm{d}}$ & $0.07^{\mathrm{d}}$ \\
\hline $\begin{array}{l}\text { Alimentado con la SOMI y el } \mathrm{NH}_{4} \mathrm{NO}_{3} \\
100 \% \text { (Control relativo) }\end{array}$ & $30.1^{\mathrm{d}}$ & $15.7^{\mathrm{d}}$ & $1.26^{\mathrm{c}}$ & $0.66^{\mathrm{d}}$ & $0.63^{\mathrm{c}}$ & $0.09^{\mathrm{d}}$ \\
\hline $\begin{array}{l}\text { Azospirillum lipoferum más la SOMI y el } \\
\mathrm{NH}_{4} \mathrm{NO}_{3} \text { al } 50 \%\end{array}$ & $32.6^{\mathrm{c}}$ & $16.5^{\mathrm{c}}$ & $2.27^{\mathrm{c}}$ & $0.80^{\mathrm{c}}$ & $0.33^{\mathrm{d}}$ & $0.27^{\mathrm{c}}$ \\
\hline $\begin{array}{l}\text { Rhizobium etli más la SOMI y el } \mathrm{NH}_{4} \mathrm{NO}_{3} \\
\text { al } 50 \%\end{array}$ & $35^{\mathrm{b}}$ & $17.5^{\mathrm{b}}$ & $2.32^{\mathrm{b}}$ & $0.88^{\mathrm{b}}$ & $1.63^{\mathrm{b}}$ & $0.41^{\mathrm{b}}$ \\
\hline $\begin{array}{l}\text { A. lipoferum/ R. etli más la SOMI y el } \\
\mathrm{NH}_{4} \mathrm{NO}_{3} \text { al } 50 \%\end{array}$ & $38.7^{\mathrm{a}}$ & $42.2^{\mathrm{a}}$ & $5.62^{\mathrm{a}}$ & $1.96^{\mathrm{a}}$ & $1.80^{\mathrm{a}}$ & $0.75^{\mathrm{a}}$ \\
\hline
\end{tabular}

Tabla 3 Respuesta de Glycine max a la inoculación con Azospirillum lipoferum y Rhizobium etli a dosis $50 \%$ de fertilizante nitrogenado a floración

\begin{tabular}{|c|c|c|c|c|c|c|}
\hline \multirow{2}{*}{ *Glycine max en suelo } & \multirow{2}{*}{$\mathbf{A P}(\mathbf{c m})$} & \multirow{2}{*}{$\mathbf{L R}(\mathbf{c m})$} & \multicolumn{2}{|c|}{ Peso fresco $(g)$} & \multicolumn{2}{|c|}{ Peso seco (g) } \\
\hline & & & Aéreo & Radical & Aéreo & Radical \\
\hline Irrigado solo agua (control absoluto) & $33.9^{\mathrm{e}^{* *}}$ & $24.5^{\mathrm{d}}$ & $7.65^{\mathrm{d}}$ & $2.83^{\mathrm{e}}$ & $1.17^{\mathrm{e}}$ & $0.68^{\mathrm{e}}$ \\
\hline $\begin{array}{c}\text { Alimentado con SOMI y el } \mathrm{NH}_{4} \mathrm{NO}_{3} \text { al } \\
100 \% \text { (control relativo) }\end{array}$ & $39.3^{\mathrm{c}}$ & $25.7^{\mathrm{c}}$ & $9.54^{\mathrm{c}}$ & $4.50^{\mathrm{d}}$ & $1.85^{\mathrm{d}}$ & $1.04^{\mathrm{d}}$ \\
\hline $\begin{array}{l}\text { Inoculado con Azospirillum lipoferum y } \\
\text { SOMI con } \mathrm{NH}_{4} \mathrm{NO}_{3} \text { al } 50 \%\end{array}$ & $38^{\mathrm{d}}$ & $28.9^{\mathrm{b}}$ & $12.42^{\mathrm{b}}$ & $6.37^{\mathrm{c}}$ & $3.57^{\mathrm{b}}$ & $1.09^{\mathrm{c}}$ \\
\hline $\begin{array}{l}\text { Inoculado con Rhizobium etli con SOMI y } \\
\qquad \mathrm{NH}_{4} \mathrm{NO}_{3} \text { al } 50 \%\end{array}$ & $43.3^{\mathrm{b}}$ & $34^{\mathrm{a}}$ & $12.60^{\mathrm{b}}$ & $9.60^{\mathrm{b}}$ & $2.77^{\mathrm{c}}$ & $1.34^{\mathrm{b}}$ \\
\hline $\begin{array}{l}\text { Coinoculado con } A \text {. lipoferum/R. etli con } \\
\text { SOMI y } \mathrm{NH}_{4} \mathrm{NO}_{3} \text { al } 50 \%\end{array}$ & $44.7^{\mathrm{a}}$ & $33.8^{\mathrm{a}}$ & $14.33^{\mathrm{a}}$ & $12.49^{\mathrm{a}}$ & $3.90^{\mathrm{a}}$ & $2.44^{\mathrm{a}}$ \\
\hline
\end{tabular}

En relación a la biomasa $G$. max inoculada con los dos géneros y especies de BAPOCEVE se registraron: 14.33 g de PFA y 12.49 g de PFR, ambos valo- res numéricos que tuvieron diferencia estadística comparados con los $9.54 \mathrm{~g}$ de PFA y $4.50 \mathrm{~g}$ PFR de G. max empleado como CR. En tanto que G. $\max$ 
inoculada con A. lipoferum y $R$. etli mientras que G. max con la doble inoculación con las BAPOCEVE registraron $3.90 \mathrm{~g}$ de PSA y $2.44 \mathrm{~g}$ de PSR ambos valores numéricos tuvieron diferencia estadística respecto a los $1.85 \mathrm{~g}$ de PSA y 1.04 en PSR de $G$. max referido como $\mathrm{CR}$.

\section{Discusión}

Los valores numéricos reportados en la Tabla 2 basados en los datos de la fenología y biomasa de G. max con A. lipoferum y/o R. etli a nivel plántula, sugieren que el incremento de la AP y LR, está asociado con el hecho de que los dos géneros y especies de BAPOCEVE empleados, tienen capacidad genética de convertir los exudados radicales de $G$. max como los ácidos orgánicos y aminoácidos del tipo del triptófano en SUPOCEVE o fitohormonas del tipo de la auxina, que mediante la inducción de la mitosis y cariocinesis del tejido radical, favorecen una acelerada y general proliferación de un elevado número de raíces laterales, para aumentar el área de exploración de esos pelos que mejoraron la mayor absorción de la raíz por el N, lo que consecuentemente optimizo el FENI reducido al 50\% (Askary et al. 2009, Benintende et al. 2010), en ese sentido se observó que el sistema radical de G. max fue más denso debido a la constante acción fitohormonal, derivada que tanto de A. lipoferum, como $R$. etli que poseen diferente capacidad genética en la conversión de los exudados de raíces, en una amplia variedad de fitohormonas, lo que mantuvo el incremento gradual del peso fresco y seco de G. max con la doble inoculación con A. lipoferum y/o R. etli, que mostraron que este género bacteriano vive como endófito en las raíces de G. max, y que además también tienen la capacidad de convertir los metabolitos derivados de azucares de la fotosíntesis en SU101
POCEVE, o fitohormonas del tipo auxina y giberelina, ambas mejoraron la absorción radical del N, y así, lograr la máxima optimización del FENI reducido al 50\% (Armenta-Bojórquez et al. 2010). Lo que evita que el excedente de $\mathrm{N}$, cause la perdida de la reserva de la materia orgánica y que en consecuencia se pierda la productividad del suelo (Alexander 1977, Isbell et al. 2013).

Mientras que los resultados registrados en la Tabla 3 relacionados con fenología y biomasa de G. max tratada con ambos géneros de BAPOCEVE a floración, sugieren que A. lipoferum y R. etli mantuvieron e incrementaron la capacidad de transformar de manera continua y complementaria, que los compuestos orgánicos liberados en los exudados de la raíces de G. max transformaron en fitohormonas: auxina y citocinina indujeron la mayor elongación y formación de raíces laterales (Bécquer et al. 2011), las que aumentaron la capacidad de absorber y optimizar el FENI reducido al 50\%, puesto que el aspecto de las hojas y raíces de G. max no mostro ninguna deficiencia relacionada con la demanda de N (Loredo-Osti 2004, Uhrich \& Benintende 2005). Por lo anterior se concluye que A. lipoferum y/o $R$. etli géneros BAPOCEVE ejercieron un efecto positivo en el crecimiento de G. max mediante la optimización del FENI al 50\%, debido a que ambas convierten los exudados radicales de G. max en SUPOCEVE: auxina, citocinina y giberelina para la maxima absorción radical del $\mathrm{N}$ del FENI a pesar de reducirlo al 50\%. La mezcla de estos dos géneros y especies de BAPOCEVE son una opción para el cultivo de G. max, lo cual evita la hiperfertilización, sin riesgo de afectar en el sano crecimiento vegetal y si de prevenir deterioro del suelo. 


\section{Conflictos de intereses}

Los autores de este artículo, declaramos que no existe ningún conflicto de interés en la planificación, ejecución y redacción de la investigación realizada, como tampoco con aquellas personas e instituciones que la financiaron.

\section{Agradecimientos}

Al proyecto 2.7 (2018) apoyado por Coordinación de la Investigación Científica de la UMSNH, Morelia, Michoacán y BIONUTRA S.A. de C.V, Maravatío, Michoacán, México.

\section{Literatura citada}

Alexander M. Introduction to soil microbiology. $2^{\text {ed }}$, John Wiley and Sons Inc. New York; 1977. p. 100-50.

Armenta-Bojórquez AD, García-Gutiérrez C, Camacho-Báez JR, Apodaca-Sánchez MÁ, Gerardo-Montoya L, Nava-Pérez E. Biofertilizantes en el desarrollo agrícola de México. Ra Ximhai 2010; 6(1): 51-6.

Askary M, Mostajeran A, Amooaghaei R, Mostajeran M. Influence of the co-inoculation Azospirillum brasilense and Rhizobium meliloti plus 2, 4-D on grain yield and N, P, K content of Triticum aestivum (cv. Baccros and Mahdavi). Am Eurasian J Agric Environ Sci 2009; 5(3), 296-307.

Bécquer CJ, Salas B, Ávila U, Palmero LA, Nápoles JA, Ramos Y, et al. Efecto de la inoculación con rizobios procedentes de Alberta, Canadá, en sorgo (Sorghum bicolor L. Moench), en condiciones de campo. Pastos y Forrajes 2011; 34(3): 303-12.
Benintende S, Uhrich W, Herrera M, Gangge F, Sterren M, Benintende M. Comparación entre coinoculación con Bradyrhizobium japonicum y Azospirillum brasilense e inoculación simple con Bradyrhizobium japonicum en la nodulación, crecimiento y acumulación de $\mathrm{N}$ en el cultivo de soja. Agriscientia 2010; 27(2): 71-7.

Corbera-Gorotiza J, Nápoles-García MC. Efecto de la inoculación conjunta Bradyrhizobium elkanii-hongos MA y la aplicación de un bioestimulador del crecimiento vegetal en soya (Glycine max (L.). Merrill) cultivar INCASOY-27. CulTrop 2013; 34 (2):5-11.

Döbereiner J, Day DJ. Associative symbiosis in tropical grasses of microorganisms and dinitrogen fixing site. In Newton W, Nyam CJ. eds. Proceedings of the 1st. International Symposium on Nitrogen Fixation. Washington State University Press. Pullman, USA; 1974. p. 518-39.

Duke SO. Natural pesticides from plants. p. 517523. En: Janick, J. \& JE. Simon (Eds.). Advances in new crops. Timber Press. Portland, Oregon; 1990. p. 829.

García-González MM, Farías-Rodríguez R, PeñaCabriales JJ, Sánchez-Yáñez JM. Inoculación del trigo var. Pavón con Azospirillum spp. y Azotobacter beijerinckii. Terra Latinoam 2005; 23(1): 65-72.

García-Villalpando JA, Castillo-Morales A, Ramírez-Guzmán ME, Rendón-Sánchez G, LarquéSaavedra MU. Comparación de los procedimientos de Tukey, Duncan, Dunnett, HSU y Bechhofer para selección de medias. Agrociencia 2001; 35(1): 79-86.

Hernández AF. La coinoculación Glomus hoi likeBradyrhizobium japonicum en la producción de soya (Glycine max) variedad Verónica para semilla. CulTrop 2008; 29(4): 41-5. 
Isbell F, Reich PB, Tilman D, Hobbie SE, Polasky S, Binder S. Nutrient enrichment, biodiversity loss, and consequent declines in ecosystem productivity. Proc Natl Acad Sci USA 2013; 110(29): 11911-6.

Loredo-Osti C, López-Reyes L, Espinosa-Victoria D. Bacterias promotoras del crecimiento vegetal asociadas con gramíneas: Una revisión. Terra Latinoam 2004; 22(2), 225-39.

Matallana A, Montero J. Invernaderos Diseño construcción y ambientación. Ed. Mundi-prensa. Madrid, España; 2001. p. 209.

Matiru VN, Dakora FD. Potential use of rhizobial bacteria as promoters of plant growth for increased yield in landraces of African cereal crops. Afr J Biotechnol 2004; 3(1): 1-7.

Peña-Cabriales JJ, Alexander M. Reservas de carbón: sobrevivencia de Rhizobium. Rev Latinoam Microbiol 1981; 23: 50-4.
Sánchez-Yáñez JM. Breve tratado de microbiología agrícola teoría y práctica. Instituto de Investigaciones Químico Biológicas, Universidad Michoacana de San Nicolás de Hidalgo, COSUSTENTA, SA de CV, Centro de Investigación y Desarrollo del Estado de Michoacán, Morelia, Mich. México; 2007. p. 130-3.

Secretaria de Agricultura Ganadería Desarrollo Rural Pesca y Alimentación. SIAP-SAGARP. Gobierno de la Republica; 2016. Mexico.www.gob.mx.

Uhrich W, Benintende S. Aplicación de Azospirillum brasilense en cultivo de soja en coinoculación con Bradyrhizobium japonicum. Rev Cient Agropecu 2005; 9(1):71-5. 